\title{
The duality between a non-Hermitian two-state quantum system and a massless charged particle
}

\author{
Robert Botet \\ Laboratoire de Physique des Solides Bât.510, \\ CNRS UMR8502 / Université Paris-Saclay, Centre d'Orsay, F-91405 Orsay, France \\ E-mail: robert.botet@u-psud.fr
}

\section{Hiroshi Kuratsuji}

Faculty of Science and Engineering, Ritsumeikan University-BKC

Noji-Hill, Kusatsu City, 525-8577, Japan

$13 / 11 / 2018$

\begin{abstract}
We show that the equations for the dynamics of a non-Hermitian twostate quantum system are the same as the equations of motion for a massless charged particle in an electromagnetic field. Using simple analytical arguments to prove this unexpected duality between two very different domains in Physics, we further exemplify it through a case-study of polarization of light propagating in a dichroic medium with magneto-optic activity.
\end{abstract}

Keywords: non-Hermitian quantum systems, Hamiltonian dynamics, massless particles

\section{Introduction}

Any real physical system is open, in the sense that it exchanges energy or mass with its environment $[1,2]$, even if only subjected to a measurement event. In other words, a perfectly isolated system is either a theoretical ideal or an approximation of a real system. This explains why the study of the dynamics of open quantum systems became one of the fundamental topics in modern physics $[2,3,4]$.

In quantum physics, the non-conservation of the total energy of the system is generally associated with non-Hermitian Hamiltonian [5], leading to subtle behaviours and complex dynamics of the quantum systems.

Non-Hermitian Hamiltonians can be simplistically divided into two groups: Hamiltonians with complex eigenvalues, and Hamiltonians with real eigenvalues.

Dissipative quantum systems belong to the former group and this concept was introduced in the context of adiabatic measurements on metastable systems [6]. Another important approach is the "continuum shell model", developed for the study of nuclear resonant states. In this theory, the system is separated into discrete states in the 
quantum structure, and a continuum of scattering states [7]. The effective nonHermitian Hamiltonian results from Feshbach's projection scheme [8]: the real parts of the eigenvalues of the Hamiltonian correspond to the energies, while the imaginary parts give the widths of the resonance states [9]. Also in nuclear physics, the complexvalued optical model potential is used to describe reactions between two nucleons [10].

The latter group (i.e. Hamiltonians with real eigenvalues) comprises theories extending the regular quantum systems with Hermitian Hamiltonians, for instance quantum systems ruled by special complex-valued potentials with space-time reflection symmetry $(\mathcal{P} \mathcal{T}$-symmetry, where $\mathcal{P}$ denotes the parity operator and $\mathcal{T}$ the time-reversal operator) [11]. This class of Hamiltonians is part of the more general subset of the pseudo-Hermitian operators [12] (that is an operator $A$ such that $A^{\dagger}=\eta A \eta^{-1}$, with $\eta$ a Hermitian automorphism and $\dagger$ the Dirac's notation for the conjugate-transpose). The pseudo-Hermitian symmetry appears naturally as soon as real input regarding the energy spectrum is required [13].

Effective non-Hermitian Hamiltonians appear in various domains of physics. The following is a non-exhaustive list of physical examples: [12],[14],[15],[16],[17],[18],[19].

\section{Scope of the paper}

In the present work, we are interested in the non-Hermitian dynamics of two-state quantum systems, the paradigm of the simplest non-trivial quantum system [20], either intrinsically (e.g. the spin-1/2 particle), or as an approximation (e.g. the ensemble consisting of the ground state and the first excited state of a low-temperature quantum system). We then further consider general non-Hermitian Hamiltonians (including the above-cited examples).

We discovered a peculiar physical feature hidden behind two-level quantum systems, namely the two-state non-Hermitian quantum dynamics is equivalent to the motion of a massless charged particle in an electromagnetic field.

Not only is this mathematical mapping a fundamental revelation, one may also better understand the complex behaviours of non-conservative quantum systems, thanks to the much more intuitive analogy illustrated by the trajectory of a charged particle in an electromagnetic field.

In Section 3, we formulate the dynamics of the non-Hermitian two-state system in Bloch variables, subsequently deriving the analogy with the motion of a massless charged particle in Section 4. Section 5 is then devoted to a full example in Optics while Section 6 is a note about the structure of a pseudo-electromagnetic field. Possible extensions to ensembles of $N$ two-state quantum systems are discussed in Section 7 , before the Conclusion. 


\section{Bloch representation of the non-conservative quantum two-state system}

The standard description of a two-state system requires representation of the state of the system as a wavefunction, $\boldsymbol{\Psi}$, in a two-dimensional complex Hilbert space. We consider here the single-mode wavefunction.

\subsection{General dynamic equations for a two-state wavefunction}

The time-evolution of the wavefunction $\Psi$ is ruled by a first-order (in the time $t$ ) differential equation and the corresponding equation for its adjoint state, $\boldsymbol{\Psi}^{\dagger}$ :

$$
i \hbar \frac{\partial \boldsymbol{\Psi}}{\partial t}=\hat{H} \boldsymbol{\Psi}, \quad-i \hbar \frac{\partial \boldsymbol{\Psi}^{\dagger}}{\partial t}=\boldsymbol{\Psi}^{\dagger} \hat{H}^{\dagger}
$$

in which $\hat{H}$ is the Hamiltonian operator including all forms of energy (kinetic and interactions), and $\hat{H}$ may depend on time. When the system is non-conservative (i.e. when the wavefunction modulus, $|\Psi|^{2}$, is not constant with time), the Hamiltonian is non-Hermitian, $\hat{H} \neq \hat{H}^{\dagger}$, and the eigenvalues of $\hat{H}$ may take complex values [21].

Evolution equation of the form (1) is known as the non-relativistic Schrödinger equation of a two-level system, the Dirac equation in the relativistic spin- $1 / 2$ case, etc.

In other applications, the dynamics equation may be formally identical to (1) though with minor differences: the operator $\hat{H}$ is not related to any physical energy but rather, to an evolution operator, while a spatial coordinate plays the role of the time and $\hbar$ is replaced by another fundamental parameter. In Section 5, we shall examine in detail just such an example: the evolution of the polarization of an electromagnetic plane-wave propagating in an optically active medium. In this case, we apply to (1)the substitution: $\hbar=h / 2 \pi \rightarrow \lambda=\lambda / 2 \pi$ (i.e. the reduced wavelength), with the operator $\hat{H}$ corresponding to the complex dielectric tensor.

\subsection{Dynamics in the Bloch representation}

Let us consider the general $2 \times 2$ Hamiltonian in its matrix form:

$$
\hat{H}=\left(\begin{array}{ll}
h_{11} & h_{12} \\
h_{21} & h_{22}
\end{array}\right),
$$

in which the four complex coefficients $h_{11}, h_{12}, h_{21}, h_{22}$ may depend on time and space, and no symmetry is assumed.

Whether $\hat{H}$ is Hermitian or not, the Hamiltonian operator can always be expanded over the basis comprising $\hat{\sigma}_{0}$ (the $2 \times 2$ identity matrix) and $\hat{\boldsymbol{\sigma}} \equiv\left(\hat{\sigma}_{1}, \hat{\sigma}_{2}, \hat{\sigma}_{3}\right)$ (in which $\hat{\sigma}_{1}, \hat{\sigma}_{2}, \hat{\sigma}_{3}$ are the usual Pauli matrices [22]):

$$
\begin{aligned}
\hat{H} & =\kappa \hat{\sigma}_{0}+\alpha \hat{\sigma}_{1}+\beta \hat{\sigma}_{2}+\gamma \hat{\sigma}_{3} \\
& =\left(\begin{array}{cc}
\kappa+\gamma & \alpha-i \beta \\
\alpha+i \beta & \kappa-\gamma
\end{array}\right),
\end{aligned}
$$


with the complex-valued coefficients:

$$
\begin{gathered}
\kappa=\frac{h_{11}+h_{22}}{2}, \\
\alpha=\frac{h_{12}+h_{21}}{2}, \beta=i \frac{h_{12}-h_{21}}{2}, \gamma=\frac{h_{11}-h_{22}}{2} .
\end{gathered}
$$

One can also use the shortened notation:

$$
\hat{H}=\kappa \hat{\sigma}_{0}+\boldsymbol{K} \cdot \hat{\boldsymbol{\sigma}},
$$

with the 3 D vector: $\boldsymbol{K}=(\alpha, \beta, \gamma) \in \mathbb{C}^{3}$.

It is worth noting that $\boldsymbol{K}^{2}=\left(E_{+}-E_{-}\right)^{2} / 4$, with $E_{ \pm}$the two eigenvalues of the Hamiltonian (3). When $\hat{H}$ is non-Hermitian, these eigenvalues may take complex values.

In general, the state of the system can be fully represented in terms of the Bloch vector, i.e. using the set of the expectation values, $S_{j}=\boldsymbol{\Psi}^{\dagger} \hat{\sigma}_{j} \boldsymbol{\Psi}$, for the four Pauli operators $\left\{\hat{\sigma}_{j}\right\}_{j=0,1,2,3}$. Due to the identity $\hat{\sigma}_{j}^{2}=\hat{\sigma}_{0}$, the four real parameters $S_{0}, S_{1}, S_{2}, S_{3}$ are not independent, and the identity:

$$
S_{1}^{2}+S_{2}^{2}+S_{3}^{2}=S_{0}^{2}
$$

remains valid at any time for a single-mode wave, even if the system is not conservative.

From (1), the time evolution of these parameters is given by the four equations:

$$
i \hbar \frac{d S_{j}}{d t}=\Psi^{\dagger}\left(\hat{\sigma}_{j} \hat{H}-\hat{H}^{\dagger} \hat{\sigma}_{j}\right) \boldsymbol{\Psi}
$$

Using the representation (3) and the identity: $(\mathbf{a} \boldsymbol{\sigma})(\mathbf{b} \boldsymbol{\sigma})=(\mathbf{a b}) \hat{\sigma}_{0}+i(\mathbf{a} \times \mathbf{b}) \boldsymbol{\sigma}$, the equations (6) may be written under the form:

$$
\begin{aligned}
i \hbar \frac{d S_{0}}{d t} & =\left(\kappa-\kappa^{\star}\right) S_{0}+\left(\boldsymbol{K}-\boldsymbol{K}^{\star}\right) \mathbf{S}, \\
i \hbar \frac{d \mathbf{S}}{d t} & =\left(\kappa-\kappa^{\star}\right) \mathbf{S}+\left(\boldsymbol{K}-\boldsymbol{K}^{\star}\right) S_{0}-i \mathbf{S} \times\left(\boldsymbol{K}+\boldsymbol{K}^{\star}\right),
\end{aligned}
$$

with $\mathbf{S}=\left(S_{1}, S_{2}, S_{3}\right)$ the unnormalized Bloch vector of the two-state system [23]. Using (8), relations (7) and (5) are equivalent. Moreover, if the Hamiltonian is Hermitian that is: $\kappa$ and $\boldsymbol{K}$ have real values - the system is conservative. Consequently $S_{0}=$ constant.

Let us introduce the real coefficient $\Gamma$ (named below: damping coefficient), and the two real-valued $3 \mathrm{D}$ vectors $\mathcal{E}$ and $\mathcal{B}$ (named below: pseudo-electric and pseudo-magnetic vectors, respectively), defined as:

$$
\begin{aligned}
\kappa & =\frac{\hbar}{2}\left(\kappa_{0}-i \Gamma\right), \\
\boldsymbol{K} & =\frac{i \hbar}{2} q(\mathcal{E}+i c \mathcal{B}),
\end{aligned}
$$


in which $q$ is a real parameter of constant value that we will arbitrarily take as positive, and $c$ is the velocity of light in vacuum (we have to select this value of $c$ in order to map the field $(\mathcal{E}, \mathcal{B})$ onto an electromagnetic field in the next Section). The combination $\mathcal{E}+i c \mathcal{B}$ written in $(9)$ becomes reminiscent of the fundamental Silberstein-Majorana representation of a real electromagnetic field [24].

We can then write the explicit forms of the pseudo-electric and pseudo-magnetic fields in terms of the initial Hamiltonian:

$$
\mathcal{E}=\frac{i}{\hbar q}\left(\begin{array}{l}
\alpha^{\star}-\alpha \\
\beta^{\star}-\beta \\
\gamma^{\star}-\gamma
\end{array}\right) ; c \mathcal{B}=-\frac{1}{\hbar q}\left(\begin{array}{l}
\alpha^{\star}+\alpha \\
\beta^{\star}+\beta \\
\gamma^{\star}+\gamma
\end{array}\right)
$$

The vector $\mathcal{B}$ represents essentially the Hermitian part of the Hamiltonian, and $\mathcal{E}$ the non-Hermitian part.

A general type of non-Hermitian Hamiltonian is encountered in the context of open quantum systems in contact with a decohering and dissipative environment. For such a system, non-equilibrium quantum dynamics result from a Hamiltonian composed of two terms $[25,26]$ :

$$
\hat{H}=\hat{H}_{+}-i \hat{\Gamma}
$$

in which both operators $\hat{H}_{+}$(the Hamiltonian of the isolated quantum system) and $\hat{\Gamma}$ (the decay rate operator) are Hermitian [26]. If the quantum system is two-level, (10) implies that the pseudo-magnetic field is only related to the Hermitian operator $\hat{H}_{+}$. Likewise, the pseudo-electric field is only related to the anti-Hermitian operator $-i \hat{\Gamma}$, in agreement with the remark written after Equ.(10).

\subsection{Lorentz invariants of a pseudo-electromagnetic field}

The scalar electromagnetic Lorentz invariants, $\mathcal{L}_{1} \equiv \mathcal{E}^{2}-c^{2} \mathcal{B}^{2}$ and $\mathcal{L}_{2} \equiv c \mathcal{E} \mathcal{B}$, of a pseudo-electromagnetic field are directly related to $\boldsymbol{K}$, since they are proportional to the real and the imaginary parts of $\boldsymbol{K}^{2}$ :

$$
\boldsymbol{K}^{2}=-\frac{\hbar^{2} q^{2}}{4}\left(\mathcal{L}_{1}+2 i \mathcal{L}_{2}\right)
$$

As a consequence: if the field $(\mathcal{E}, \mathcal{B})$ turns out to be a real electromagnetic field, then $\boldsymbol{K}^{2}$ (which is essentially the squared difference of the eigenvalues of the Hamiltonian $\hat{H}$ ) is Lorentz-invariant.

In addition, it is clear from (12) that the condition $\mathcal{L}_{2}=0$ is a necessary condition for the eigenvalues of $\hat{H}$ be real. This remark will be used in Section 5 when we discuss cases where the pseudo-electric field and the pseudo-magnetic field are orthogonal each other. 


\subsection{The dynamic equations for the Bloch vector}

Let us now write the evolution equations of the Bloch vector in terms of the pseudoelectromagnetic field. Using the definitions of $\kappa$ and $\boldsymbol{K}$ as: $i\left(\kappa-\kappa^{\star}\right)=\hbar \Gamma, i\left(\boldsymbol{K}-\boldsymbol{K}^{\star}\right)=$ $-\hbar q \mathcal{E}$ and $\left(\boldsymbol{K}+\boldsymbol{K}^{\star}\right)=-\hbar q c \mathcal{B}$, equations (7)-(8) can be rewritten as:

$$
\begin{aligned}
\frac{d S_{0}}{d t} & =q(\mathbf{S} \cdot \mathcal{E})-\Gamma S_{0}, \\
\frac{d \mathbf{S}}{d t} & =q\left(S_{0} \mathcal{E}+c \mathbf{S} \times \mathcal{B}\right)-\Gamma \mathbf{S} .
\end{aligned}
$$

One then notices in these equations that the parameter $\Gamma$ appearing in (13)-(14) is essentially the inverse of a relaxation time, in the Bloch sense [27]. In particular, when $\Gamma \neq 0$ and the pseudo-electromagnetic field vanishes, [i.e. $(\mathcal{E}, \mathcal{B})=(0,0)$, the full solution of (13)-(14) becomes:

$$
\begin{gathered}
S_{0}(t)=S_{0}(0) e^{-\bar{\Gamma} t} ; \mathbf{S}(t)=\mathbf{S}(0) e^{-\bar{\Gamma} t} \\
\bar{\Gamma}=\frac{1}{t} \int_{0}^{t} \Gamma\left(t^{\prime}\right) d t^{\prime}
\end{gathered}
$$

where the real parameter $\bar{\Gamma}$ is equal to $\Gamma$ when $\Gamma$ does not depend on the time. Dissipation imposes that $\Gamma(t)>0$, resulting in $\bar{\Gamma}>0$.

A way to remove the coefficient $\Gamma$ from the evolution equations is to consider the normalized Bloch vector $\mathbf{n}=\mathbf{S} / S_{0}$ (unitary because of (5)). Using this auxiliary quantity, equation (14) reads:

$$
\frac{d \mathbf{n}}{d t}=q \mathbf{n} \times(c \mathcal{B}-\mathbf{n} \times \mathcal{E})
$$

characterizing the quantum state of system's evolution as the motion of the point $\mathbf{n}=(\sin \theta \cos \phi, \sin \theta \sin \phi, \cos \theta)$ expressed in spherical coordinates on the unit Bloch sphere. This description is related to the spinor representation of a two-state system $\ddagger$.

\section{The analogy with the motion of a massless, charged particle}

In the identity (12), we defined the two electromagnetic Lorentz invariants, $\mathcal{L}_{1}, \mathcal{L}_{2}$, as if $(\mathcal{E}, \mathcal{B})$ were a true electromagnetic field. These invariants also appear naturally in the evolution equation of the Bloch vector. For example, writing the equations (13)(14) under the matrix form: $d\left(S_{0}, S_{1}, S_{2}, S_{3}\right) / d t=M\left(S_{0}, S_{1}, S_{2}, S_{3}\right)$, the characteristic polynomial of the $4 \times 4$ real matrix $M$ is found to be: $(X+\Gamma)^{4}+\mathcal{L}_{1}(X+\Gamma)^{2}-\mathcal{L}_{2}^{2}$. It means that the dynamics of the non-Hermitian two-state system is entirely determined by the three scalar quantities: $\Gamma, \mathcal{L}_{1}, \mathcal{L}_{2}$.

$\ddagger$ The case of the $n$-state quantum system, with $n \geq 3$, can be studied similarly using $2 n-1$ real Bloch components. However, theory of electromagnetism in the $2 n-1$-dimensional space, e.g. [50], is not as limpid as in the 3-dimensional case 
Let us then consider the relativistic 4-momentum, $\mathbf{P}$, of a virtual particle of 3momentum $\boldsymbol{p}$ and total energy $E$, according to the following expression:

$$
\mathbf{P} \equiv\left(\frac{E}{c}, \boldsymbol{p}\right)=e^{\bar{\Gamma} t}\left(S_{0}, S_{1}, S_{2}, S_{3}\right)
$$

in which $\bar{\Gamma}$ is defined as in (15). Although scaling by $\exp (\bar{\Gamma} t)$ in (17) is not compulsory, it helps reduce the equations of motion below to a simpler form. If we do not consider the scaling, then a dissipative force [28] must be added.

Let us also introduce the temporal variable $\tau$ defined as:

$$
d \tau=\frac{E}{c} d t
$$

Clearly, $\tau$ is an increasing function of time $t$ since the energy $E$ is real and positive.

Thanks to definitions (17) and (18), the state-evolution equations (13)-(14) now become:

$$
\begin{aligned}
& \frac{d E}{d \tau}=q \mathcal{E} \cdot \mathbf{v}, \\
& \frac{d \boldsymbol{p}}{d \tau}=q(\mathcal{E}+\mathbf{v} \times \mathcal{B}),
\end{aligned}
$$

where we introduce the 3-velocity, $\mathbf{v}$, of the virtual particle such that: $\mathbf{v} / c=\mathbf{n}=\mathbf{S} / S_{0}$ (since: $\boldsymbol{p}=E \mathbf{v} / c^{2}$ for a relativistic particle [29]). In particular, the magnitude of the velocity is here a constant: $\mathbf{v}^{2}=c^{2}$, resulting from the identity $\mathbf{S}^{2}=S_{0}^{2}$.

Amazingly, the equations (19)-(20) are exactly the same as the equations of motion of a charged (charge: $q$ ), massless ( Lorentz force due to the external pseudo-electromagnetic field $(\mathcal{E}, \mathcal{B})$. We henceforth denote such a virtual MassLess, Charged, particle as a 'MLC particle'.

To complete this case-study, let us give the time-evolution equation for the particle's velocity. The corresponding nonlinear equation derived either from (20)-(19) or from (16), is:

$$
\frac{d \mathbf{v}}{d t}=q c\left(\mathcal{E}+\mathbf{v} \times \mathcal{B}-\frac{\mathbf{v}}{c}\left(\frac{\mathbf{v}}{c} \cdot \mathcal{E}\right)\right)
$$

We therefore find the usual expression for the acceleration of a MLC particle propagating in an electromagnetic field $(\mathcal{E}, \mathcal{B})[30]$.

To summarize, we showed in (13)-(14) and (19)-(20), that the time-evolution of the normalized Bloch vector $\mathbf{S} / S_{0}$ of a non-conservative two-state quantum system ruled by the non-Hermitian Hamiltonian $\hat{H}$, is identical to the $\tau$-evolution of the reduced velocity $\mathbf{v} / c$ of a massless charged particle in a pseudo-electromagnetic field $(\mathcal{E}, \mathcal{B})$.

The variables $\tau$ and $t$ are related through the equation $c d \tau=E d t$, and the realvalued pseudo-electromagnetic field $(\mathcal{E}, \mathcal{B})$ is related to the Hamiltonian $\hat{H}$ through the 
equation: $\hat{H}=(\hbar / 2)\left[\kappa_{0} \hat{\sigma}_{0}-q c \mathcal{B} \hat{\boldsymbol{\sigma}}+i\left(-\Gamma \hat{\sigma}_{0}+q \mathcal{E} \hat{\boldsymbol{\sigma}}\right)\right]$.

Although a spinless MLC particles does not appear to exist in the real world, we do not know of any strong reasons forbidding its existence [31], and many works have been devoted to the dynamics of this hypothetical particle [32].

An advantage of establishing this equivalence between a non-Hermitian quantum system and MLC particle dynamics is that the movement of a charged particle in an electromagnetic field is intuitive to understand, whereas the comprehension of open quantum system dynamics - even if two-state -, is not often immediately apparent.

\section{An example: the constant crossed pseudo-electromagnetic field}

We discuss hereafter the case where the second Lorentz invariant, $\mathcal{L}_{2}$ as defined in (12), vanishes. This means that the pseudo-electric and -magnetic fields are orthogonal, since $\mathcal{L}_{2}=c \mathcal{E} \mathcal{B}=0$. This situation is usual when the eigenvalues of the Hamiltonian are required to take real values (since: $\mathcal{L}_{1} \leq 0 ; \mathcal{L}_{2}=0$ from the relation (12) in this case). But instead, we shall consider here a more general situation in which the eigenvalues of the non-Hermitian Hamiltonian may be complex.

Let us consider the case of the MLC particle in a constant crossed electromagnetic field:

$$
\mathcal{E}=\mathcal{E}\left(\begin{array}{l}
1 \\
0 \\
0
\end{array}\right) ; \mathcal{B}=\mathcal{B}\left(\begin{array}{l}
0 \\
0 \\
1
\end{array}\right)
$$

in which $\mathcal{E}$ and $\mathcal{B}$ are the constant amplitudes of the pseudo-electric and pseudo-magnetic fields, respectively. Without loss of generality, we choose the direction of the $z$-axis such that $\mathcal{B}>0$.

In order to ascertain the trajectory of the MLC particle, we also need to define its initial state using its position, $\mathbf{x}(0)$, and initial velocity, $\mathbf{v}(0)$.

$$
\mathbf{x}(0)=\left(\begin{array}{l}
0 \\
0 \\
0
\end{array}\right) ; \mathbf{v}(0)=\left(\begin{array}{l}
c \\
0 \\
0
\end{array}\right) \text {. }
$$

To simplify the present demonstration, we have set the initial particle velocity to be parallel to the pseudo-electric field, but this is by no means a necessary constraint; more general initial conditions simply lead to more complicated expressions for the particle trajectory. 
5.1. Examples of crossed pseudo-electromagnetic fields and a special optical system case-study.

A generic example of a quantum system that manifests a crossed pseudo-electromagnetic field is a two-level system coupled to a dissipative environment such that the total non-Hermitian Hamiltonian of the system is the sum of a Hermitian part and of an anti-Hermitian part [33]:

$$
\mathcal{H}=-\hbar \Omega \hat{\sigma}_{1}-i \hbar \Omega\left(\gamma \hat{\sigma}_{0}+a_{2} \hat{\sigma}_{2}+a_{3} \hat{\sigma}_{3}\right)
$$

with $\Omega, \gamma, a_{2}, a_{3}$ four real coefficients. Using the definitions presented in (9), one obtains readily the pseudo-electromagnetic field under the form: $q \mathcal{E}=-2 \Omega\left(0, a_{2}, a_{3}\right)$, $q c \mathcal{B}=2 \Omega(1,0,0)$ for which the Lorentz invariant $\mathcal{L}_{2}$ vanishes, (i.e. $\mathcal{E}$ and $\mathcal{B}$ are orthogonal. An important case discussed in [33, 34, 35] is $a_{2}=0$. In this case, the pseudo-electromagnetic field is found to be similar to (22) (except for a spatial rotation of $\pi / 2$ around the $y$-axis), with the coefficients $\mathcal{E}=-2 \Omega a_{3} / q, \mathcal{B}=2 \Omega / q c$, while the damping coefficient is $\Gamma=2 \Omega \gamma$.

A concrete example of this type of quantum system is the direct photodetection of fluorescent photons emitted by a two-level atom [34]. In this case, the electromagnetic field acts as a quantum probe [2].

Another instance of crossed pseudo-electromagnetic field is found in the context of the operators with $\mathcal{P} \mathcal{T}$-symmetry. We take the example proposed in [36]:

$$
\hat{H}=\left(\begin{array}{cc}
r+t \cos \phi-i s \sin \phi & i s \cos \phi+t \sin \phi \\
i s \cos \phi+t \sin \phi & r-t \cos \phi+i s \sin \phi
\end{array}\right)
$$

in which the coefficients $s$ and $t$ are real and such that: $|s| \leq|t|$ for the energy spectrum to take real values. Consequently, the definitions given in (9) would lead to a pseudoelectromagnetic field under the form:

$$
\frac{\hbar}{2} q \mathcal{E}=s\left(\begin{array}{c}
\cos \phi \\
0 \\
-\sin \phi
\end{array}\right) ; \frac{\hbar}{2} q c \mathcal{B}=-t\left(\begin{array}{c}
\sin \phi \\
0 \\
\cos \phi
\end{array}\right)
$$

with the orthogonality property: $\mathcal{L}_{2}=c \mathcal{E B}=0$. Unsurprisingly, the condition of real-valued energies (given by $|s| \leq|t|$ ) results in the inequality $\mathcal{L}_{1} \equiv \mathcal{E}^{2}-c^{2} \mathcal{B}^{2}=$ $(2 / \hbar q)^{2}\left(s^{2}-t^{2}\right) \leq 0$, such that $\boldsymbol{K}^{2} \geq 0$ because of (12).

If $\phi=0$, then we find the exact condition described in (22). In the more general case where $\phi \neq 0$, it is clear from (26) that this corresponds to a simple rotation of the pseudo-electromagnetic field by the angle $\phi$ around the $y$-axis.

We now consider a whole other situation corresponding to the following optical system: a dichroic, birefringent, dielectric medium with $x, y, z$ the principal axes 
[37]. A constant external magnetic field, $\boldsymbol{H}=(0,0, H)$, is applied to the opticallyactive medium. We seek to examine the circular polarization of a monochromatic electromagnetic plane wave of frequency $\omega$, propagating along the $z$-direction. This problem has been studied within the context of the non-Hermitian matrices in [38].

The electric induction, $\mathbf{D}$, of the wave is related to its electric field $\mathbf{E}$ by the constitutive relation [39]:

$$
\mathbf{D}=\epsilon \mathbf{E}+i f \mathbf{H} \times \mathbf{E},
$$

in which the first term results in dichroism and the second term in the Faraday effect. The magneto-optic parameter $f$ is real and is characteristic of the medium. The magnetic permeability of the medium is expected to be $\mu_{0}$. In the basis $(x, y, z)$, the dielectric tensor expresses as:

$$
\epsilon=\left(\begin{array}{ccc}
\epsilon_{0}+i \kappa_{x} & 0 & 0 \\
0 & \epsilon_{0}+i \kappa_{y} & 0 \\
0 & 0 & \epsilon_{0}+i \kappa_{z}
\end{array}\right)
$$

where the constitutive coefficients $\kappa_{x}, \kappa_{y}, \kappa_{z}$ are positive and proportional to the respective absorption coefficients along the three axes.

Within the envelope approximation, the evolution of the wave polarization can be written as a two-state Schrödinger-like equation [40]. Indeed, the spatial part of the electric field of the wave is written: $\mathbf{E}=\mathbf{F} \exp \left(i k_{0} z\right)$, in which $k_{0}=\omega / c$ is the wavenumber in vacuum. The spatial variations of the envelope function, $\mathbf{F}=\left(F_{x}, F_{y}, 0\right)$, are expected to be much slower than the fast-oscillating term $\exp \left(i k_{0} z\right)$ (envelope approximation). Then, neglecting $d^{2} \mathbf{F} / d z^{2}$ in front of the $d \mathbf{F} / d z$ terms in the Maxwell equations, the following evolution equation is obtained:

$$
i \chi \frac{d \mathbf{F}}{d z}=\frac{1}{2 \epsilon_{0}}\left(\begin{array}{cc}
-i \kappa_{x} & \text { if } H \\
-i f H & -i \kappa_{y}
\end{array}\right) \mathbf{F} .
$$

The equation (27) is of the general form (1), with the real coordinate $z=c t$ playing the role of the time in the equation, and the reduced wavelength in vacuum, $\lambda \equiv \lambda / 2 \pi$, replaces the reduced Planck constant $\hbar$. That way, the effective Hamiltonian $\hat{H}_{\text {eff }}$ (related to the dielectric tensor) is defined. One can note that $\hat{H}_{\text {eff }}$ is not Hermitian, due to the dichroic coefficients $\kappa_{x}, \kappa_{y}$ expressing energy dissipation.

If we are interested in the circular polarization state of the wave, it is more practical to change the representation from the linear-polarization basis to the circularpolarization basis. This is achieved by considering the transformation:

$$
\left(\begin{array}{l}
F_{+} \\
F_{-}
\end{array}\right)=T\left(\begin{array}{l}
F_{x} \\
F_{y}
\end{array}\right)
$$

with $T$ the unitary matrix:

$$
T=\frac{1}{\sqrt{2}}\left(\begin{array}{cc}
1 & -i \\
1 & i
\end{array}\right)
$$


In the circular-polarization basis, the effective Hamiltonian is: $\hat{H}_{c}=T \hat{H}_{\mathrm{eff}} T^{-1}$, hence the representation:

$$
\begin{gathered}
i \rtimes \frac{d \mathbf{F}_{ \pm}}{d t}=\hat{H}_{c} \mathbf{F}_{ \pm} \\
\hat{H}_{c}=\frac{c}{2 \epsilon_{0}}\left(\begin{array}{cc}
-i\left(\kappa_{x}+\kappa_{y}\right) / 2-f H & i\left(\kappa_{y}-\kappa_{x}\right) / 2 \\
i\left(\kappa_{y}-\kappa_{x}\right) / 2 & -i\left(\kappa_{x}+\kappa_{y}\right) / 2+f H
\end{array}\right),
\end{gathered}
$$

in which we used the two-state function: $\mathbf{F}_{ \pm}=\left(F_{+}, F_{-}, 0\right)$. We note that this symmetric Hamiltonian is a particular case of the more general $\mathcal{P} \mathcal{T}$-symmetric Hamiltonian (25) in which $\phi=0$ and the coefficients $s$ and $t$ are real and respectively equal to: $s=\left(c / 2 \epsilon_{0}\right)\left(\kappa_{y}-\kappa_{x}\right) / 2$ and $t=-\left(c / 2 \epsilon_{0}\right) f H$.

Using the definition (3), one obtains readily the vector:

$$
\boldsymbol{K}=\frac{c}{2 \epsilon_{0}}\left(\begin{array}{c}
i\left(\kappa_{y}-\kappa_{x}\right) / 2 \\
0 \\
-f H
\end{array}\right)
$$

and the coefficient $\kappa=-i c\left(\kappa_{x}+\kappa_{y}\right) / 4 \epsilon_{0}$. Then, from the definition (9), the damping coefficient is: $\Gamma=c\left(\kappa_{x}+\kappa_{y}\right) /\left(4 \chi \epsilon_{0}\right)$, and the constant crossed pseudo-electromagnetic field takes on the form in $(22)$ :

$$
q \mathcal{E}=\frac{c}{4 \lambda \epsilon_{0}}\left(\kappa_{y}-\kappa_{x}\right)\left(\begin{array}{l}
1 \\
0 \\
0
\end{array}\right), q c \mathcal{B}=\frac{c}{2 \lambda \epsilon_{0}} f H\left(\begin{array}{l}
0 \\
0 \\
1
\end{array}\right) .
$$

In this representation, $q \mathcal{E}$ and $q c \mathcal{B}$ both possess the dimension of the inverse of a characteristic time.

\subsection{Case of a constant pseudo-magnetic field alone}

If $\mathcal{E}=0$ and $\Gamma=0$ (that is the conservative case), the total energy, E, of the MLC particle is constant. The equation (21) for the particle velocity becomes:

$$
\frac{d \mathbf{v}}{d t}=q c \mathbf{v} \times \mathcal{B}
$$

Its solution, consistent with the initial conditions (23), is:

$$
\frac{v_{x}}{c}=\cos \left(\Omega_{0} t\right) ; \frac{v_{y}}{c}=-\sin \left(\Omega_{0} t\right),
$$

with the frequency:

$$
\Omega_{0}=q c \mathcal{B}
$$

The trajectory of the particle is then simply a circle of radius $2 / q \mathcal{B}$ in the plane $z=0$ :

$$
x=\frac{2 c}{\Omega_{0}} \sin \left(\Omega_{0} t / 2\right) \cos \left(\Omega_{0} t / 2\right) ; y=-\frac{2 c}{\Omega_{0}} \sin ^{2}\left(\Omega_{0} t / 2\right) ; z=0 .
$$


Subsequently, let us note that, in this non-dissipative case, the shortest time, $t_{p}(0)$, for the particle to reverse the direction of its velocity $\left(v_{x}=+c \rightarrow v_{x}=-c\right)$ is:

$$
t_{p}(0)=\frac{\pi}{\Omega_{0}} .
$$

This is the passage time as defined in [41].

This case corresponds to the pure Faraday effect, without any dichroic feature. From solution (32), the Stokes vector, $\left(S_{1} / S_{0}, S_{2} / S_{0}, S_{3} / S_{0}\right)$, is rotating around the $S_{3^{-}}$ axis with constant temporal frequency $\Omega_{0}$ on the Poincare sphere (the analogue of the Bloch sphere for optics polarization problems).

In terms of the optical system, the initial condition $\left(S_{1}(0)=1\right)$ is the linearlypolarized wave with $x$ the polarization direction. Hence, the wave remains linearly polarized (since $S_{3}(t)=0$ ) with a regular rotation of its polarization plane. Indeed, the Faraday polarization angle is: $\varphi_{F}=-\Omega_{0} t / 2=\left(f / 4 \lambda \epsilon_{0}\right) H z\left(\right.$ since $S_{2}(t) / S_{1}(t)=$ $\left.-\tan \Omega_{0} t\right)$. The time $t_{p}(0)$ is then the shortest time for the linear-polarization angle of the wave to rotate by $\pi / 2$.

\subsection{The general case of a constant crossed electromagnetic field}

In the general case where $\mathcal{E} \neq 0$ and $\mathcal{B} \neq 0$, the 3 -acceleration of MLC particle is always orthogonal to its 3 -velocity $\mathbf{v}$, and it is written as (see Equ.(21)):

$$
\frac{d \mathbf{v}}{d t}=q \mathbf{v} \times\left(c \mathcal{B}-\frac{\mathbf{v}}{c} \times \mathcal{E}\right) .
$$

If, at a given time $t, \mathbf{v}(t)$ lies in the plane $(x, y)$, the acceleration $d \mathbf{v} / d t$ is in the plane $(x, y)$ too, since both vectors $\mathcal{B}$ and $\mathbf{v} \times \mathcal{E}$ are along $z$. One deduces that for an initial velocity $\mathbf{v}(0)$ along the $x$-axis, the whole trajectory of the particle for $t>0$ is planar and contained in the $(x, y)$ plane.

It is now simple to write explicitly equation (36) for the components $v_{x}$ and $v_{y}$ (since $v_{z}=0$ for the selected initial condition), using the expressions of the pseudoelectromagnetic field (22):

$$
\begin{aligned}
\frac{1}{\Omega_{0}} \frac{d v_{x}}{d t} & =\left(1+\frac{\mathcal{E}}{\mathcal{B} c^{2}} v_{y}\right) v_{y} \\
\frac{1}{\Omega_{0}} \frac{d v_{y}}{d t} & =-\left(1+\frac{\mathcal{E}}{\mathcal{B} c^{2}} v_{y}\right) v_{x}
\end{aligned}
$$

with $\Omega_{0}$ given by (33).

We then introduce the angle $\theta$ between the direction of the velocity and the direction of the pseudo-electric field, namely: $v_{x}=c \cos \theta, v_{y}=c \sin \theta$. The equations (37)-(38) shorten into a single equation in $\theta$ :

$$
\frac{d \theta}{d\left(\Omega_{0} t\right)}=-\left(1+\frac{\mathcal{E}}{c \mathcal{B}} \sin \theta\right)
$$


which is easy to solve. The form of this equation's solution depends essentially on the value of the parameter $\mathcal{E} / c \mathcal{B}$.

- the case of a small pseudo-electric field case: $|\mathcal{E}|<c \mathcal{B}$ (i.e. $\mathcal{L}_{1}<0$ )

The solution of (37)-(38) (including the initial condition $\theta=0$ for $t=0$ ) leads to the particle velocity:

$$
\begin{aligned}
\frac{v_{x}}{c} & =\frac{\cos (\Omega t-\psi) \cos \psi}{1+\sin (\Omega t-\psi) \sin \psi}, \\
\frac{v_{y}}{c} & =-\frac{\sin (\Omega t-\psi)+\sin \psi}{1+\sin (\Omega t-\psi) \sin \psi},
\end{aligned}
$$

in which the frequency $\Omega$ and phase $\psi$ are given by the expressions:

$$
\begin{aligned}
\sin \psi & =\frac{\mathcal{E}}{c \mathcal{B}}, \\
\Omega & =q \sqrt{c^{2} \mathcal{B}^{2}-\mathcal{E}^{2}}=\Omega_{0} \cos \psi .
\end{aligned}
$$

As in the previous Section, the passage time, $t_{p}$, is defined as the smallest positive time for which: $v_{x}=-c$. One finds:

$$
\begin{aligned}
t_{p}(\mathcal{E}) & =\frac{\pi-2|\psi|}{\Omega} \\
& =\frac{2}{q c \mathcal{B}} \frac{\arctan \sqrt{c^{2} \mathcal{B}^{2} / \mathcal{E}^{2}-1}}{\sqrt{1-\mathcal{E}^{2} / c^{2} \mathcal{B}}},
\end{aligned}
$$

generalizing the relation (35) to the non-Hermitian case $\mathcal{E} \neq 0$.

The result (44) is a particular case of a more general formula giving the value of the passage time $t_{p}$ for two-dimensional non-Hermitian Hamiltonian with constant coefficients [42]. Using the notations as in (3), the general expression for the passage time is:

$$
t_{p}=\left|\frac{i}{2 K} \log \left(\frac{\alpha-K}{\alpha+K}\right)\right|,
$$

where $K=\sqrt{\alpha^{2}+\beta^{2}+\gamma^{2}}$ is real [43]. Then, using (9) and (22) in (46), one recovers (45).

The main conclusion of [42] holds true in the present case: in the non-Hermitian Hamiltonian case (where $\mathcal{E} \neq 0$ ), the value of the minimum time (45) to transform a given polarization state into its orthogonal counterpart is always smaller than for the Hermitian case (35). In other words, evolution is faster in non-Hermitian than in Hermitian quantum mechanics [42].

One should notice that the problem of the minimal passage time in non-Hermitian system can be tackled here in a new way, using the equivalence with the motion of a massless charged particle. The question then transposes into finding the shortest particle trajectory in the velocity space using a variational principle [44]. With this 
approach, equation (21) plays the key role in calculating the passage time.

In the corresponding optical system, the solution (40)-(41) implies that the traveling wave is linearly polarized, and that the wave polarization plane is rotating nonuniformly, with the spatial period $\Omega_{0} \cos \psi / c$. To be precise, the polarization angle, $\varphi_{F}$, is such that:

$$
-\tan \left(2 \varphi_{F}\right)=\frac{\tan (\Omega z / c-\psi)}{\cos \psi}+\frac{\tan \psi}{\cos (\Omega z / c-\psi)},
$$

with the value of $\psi$ given by (42), and $\Omega$ by (43).

The trajectory of the particle is found analytically from time-integration of equations (40)-(41) and the use of initial conditions per (23) [45]. An example of trajectory is given in Fig.1.

- the cse of a large pseudo-electric field case: $|\mathcal{E}|>c \mathcal{B}$ (i.e. $\mathcal{L}_{1}>0$ )

When the pseudo-electric field (the non-Hermitian terms) is dominant, the energies take on complex values. The particle trajectory no longer exhibits spatial oscillations. Instead of trigonometric functions, as in (40)-(41), hyperbolic functions must be used [33] after replacing $\Omega$ by $i q \sqrt{\mathcal{E}^{2}-c^{2} \mathcal{B}^{2}}$.

From the general differential equations (37)-(38), one derives a simple result: for larger times, the trajectory sticks to the special stationary solution:

$$
\frac{v_{x}}{c}= \pm \sqrt{1-\frac{c^{2} \mathcal{B}^{2}}{\mathcal{E}^{2}}} ; \frac{v_{y}}{c}=-\frac{c \mathcal{B}}{\mathcal{E}} ; \frac{v_{z}}{c}=0
$$

with $d \mathbf{v} / d t=0$. The sign of $v_{x}$ is determined by the initial conditions.

For the corresponding optical problem, this means that the Stokes vector tends to the fixed point given by (47), after replacing $v_{x} / c \rightarrow S_{1} / S_{0}, v_{y} / c \rightarrow S_{2} / S_{0}$. In other words, after traveling a long distance in the magneto-optical medium, the wave becomes linearly polarized with a stable angle, $\varphi_{F}$, between the polarization plane and the initial polarization plane, and: $\sin 2 \varphi_{F}= \pm c \mathcal{B} / \mathcal{E}$ (the sign of which depends on the initial condition).

- the "exceptional point": $|\mathcal{E}|=c \mathcal{B}$ (i.e. $\mathcal{L}_{1}=\mathcal{L}_{2}=0$ )

The threshold between the oscillating behaviour and the fixed point occurs for $|\mathcal{E}|=c \mathcal{B}$ (an example is shown on the Fig.1). In the terminology of non-Hermitian systems, this condition is called the "exceptional point" [46]. The complete solution of the dynamics with the initial conditions $v_{x}(0)=c, v_{y}(0)=0$ is therefore:

$$
\frac{v_{x}}{c}=\frac{2\left(1+\Omega_{0} t\right)}{\left(1+\Omega_{0} t\right)^{2}+1} ; \frac{v_{y}}{c}=\frac{\left(1+\Omega_{0} t\right)^{2}-1}{\left(1+\Omega_{0} t\right)^{2}+1} ; \frac{v_{z}}{c}=0
$$

It is clear that the velocity $v_{x}$ cannot attain negative values, so $t_{p}$ is no longer defined.

The limit values (48) are recovered, and the angle of the limit polarization plane with the initial polarization plane is $\pi / 4$. 


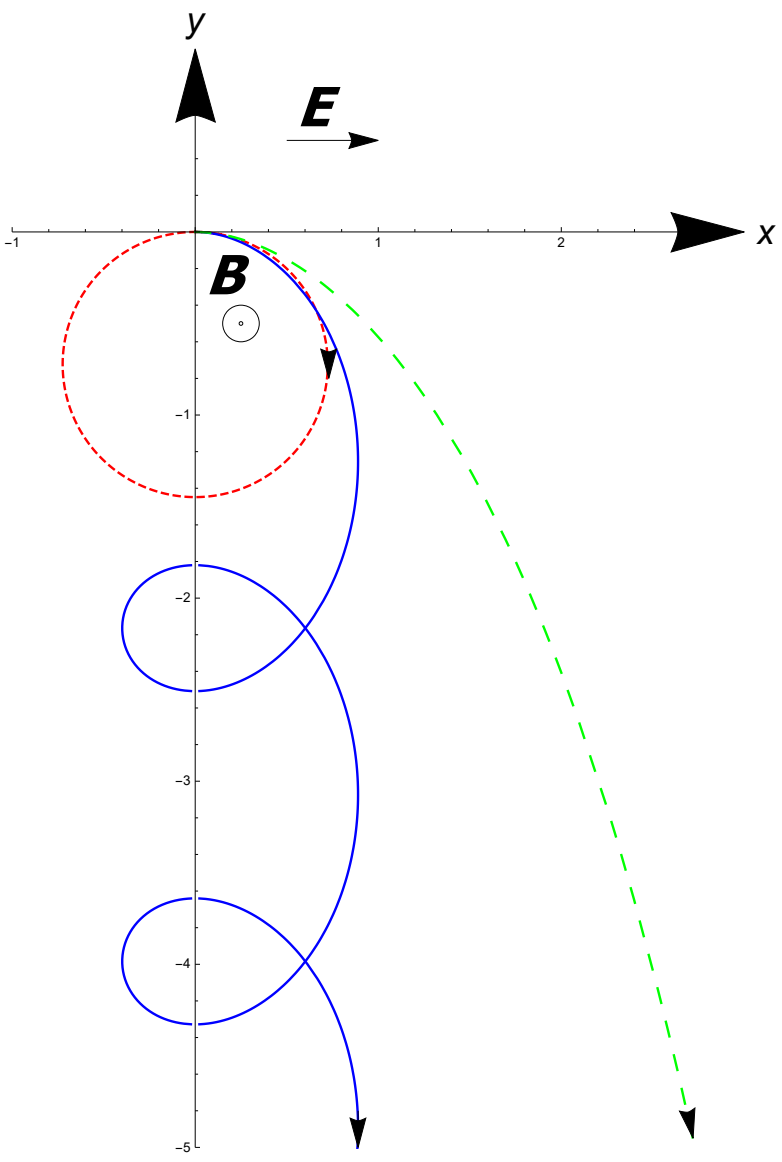

Figure 1. Three examples of trajectories of a MLC particle with charge $q=1$, in a constant crossed pseudo-electromagnetic field. The dashed red circle is the Hermitian case $\mathcal{E}=(0,0,0) ; c \mathcal{B}=(0,0,29 / 21)$; the blue continuous curve is the non-Hermitian case $\mathcal{E}=(20 / 21,0,0) ; c \mathcal{B}=(0,0,29 / 21) ;$ the green dashed curve is the exceptional point $\mathcal{E}=(29 / 21,0,0) ; c \mathcal{B}=(0,0,29 / 21)$. In all three cases, the initial conditions are: $\mathbf{x}(0)=(0,0,0) ; \mathbf{v}(0) / c=(1,0,0)$. Unlike for a massive particle, the MLC particle trajectory experiences a drift along the $y$-axis in the case of the non-vanishing pseudo-electric field $\mathcal{E}$ along the $x$-direction, because the acceleration generated by $\mathcal{E}$ is perpendicular to this field. The polarization state of the corresponding optical system, here an electromagnetic plane wave subject to Faraday effect in a dichroic medium, is the direction of the particle velocity: $\left(S_{1}, S_{2}\right)=\left(v_{x} / c, v_{y} / c\right)$ (see text for details).

\section{A note about the pseudo-electromagnetic field}

One may be surprised to learn that the Lorentz force (20) applied to the MLC particle when the field $(\mathcal{E}, \mathcal{B})$ has nothing to do with a genuine electromagnetic field.

To clarify this point, we start from the description of the system in canonical coordinates, $\mathbf{x}_{c}$ and $\mathbf{p}_{c}$, in the phase space. With this approach, the spatial coordinates, $\mathbf{x}_{c}$, are defined as conjugates to the canonical momentum, $\mathbf{p}_{c}$, through the Hamilton equation $d \mathbf{x}_{c} / d t=\partial \mathcal{H}_{c} / \partial \mathbf{p}_{c}$, in which $\mathcal{H}_{c}$ is the canonical Hamiltonian [47]. The field $(\mathcal{E}, \mathcal{B})$ is generally a function of $\mathbf{x}_{c}$.

The total energy, $E$, and 3-momentum, $\boldsymbol{p}$, of the MLC particle, must incorporate 
the structure of the scalar and vector potentials when they exist. The minimal coupling procedure yields: $E \equiv c S_{0}=\mathcal{H}_{c}-q \Phi, \quad \boldsymbol{p}=\mathbf{p}_{c}-q \mathbf{A}$, with $q$ the "charge" coupling the particle to the potentials $(\Phi, \boldsymbol{A})$. According to the relativistic quantum field theory [48], the Hamiltonian $\mathcal{H}_{c}$ of the system can be expressed in terms of the potentials:

$$
\mathcal{H}_{c}=\sqrt{m^{2} c^{4}+\boldsymbol{p}^{2} c^{2}}+q \Phi
$$

with $m=0$, since the MLC particle is massless. The Hamilton equations:

$$
\frac{d \mathbf{p}_{c}}{d t}=-\frac{\partial \mathcal{H}_{c}}{\partial \mathbf{x}_{c}} ; \quad \frac{d \mathbf{x}_{c}}{d t}=+\frac{\partial \mathcal{H}_{c}}{\partial \mathbf{p}_{c}},
$$

then lead to (see the detailed derivation in $\S 16$ of [30]):

$$
\frac{d \boldsymbol{p}}{d t}=q\left[-\left(\frac{d \boldsymbol{A}}{d t}-(\mathbf{v} \boldsymbol{\nabla}) \boldsymbol{A}\right)-\boldsymbol{\nabla} \Phi+\mathbf{v} \times(\boldsymbol{\nabla} \times \boldsymbol{A})\right] .
$$

Denoting: $\mathcal{E} \equiv-\partial \boldsymbol{A} / \partial t-\boldsymbol{\nabla} \Phi$ and $\mathcal{B} \equiv \boldsymbol{\nabla} \times \boldsymbol{A}$, the right-hand term of (50) is the regular Lorentz force: $q(\mathcal{E}+\mathbf{v} \times \mathcal{B})$ without having to invoke the Maxwell equations [49].

\section{Possible extensions}

In this Section, we wish to address briefly possible generalizations of our approach, extending it to an ensemble of $N$ two-level quantum systems.

7.1. Statistics of an ensemble of $N$ incoherent, identical, two-state quantum systems with non-Hermitian Hamiltonian

We consider, in this Section, a large number, $N$, of two-state quantum systems ruled by the same non-Hermitian Hamiltonian. This ensemble is supposed to be incoherent, i.e. the state of any individual system does not depend on the states of the other systems.

The quantum state of this statistical ensemble is generally a mixed state which can be described by the averaged values of the Bloch parameters:

$$
\begin{aligned}
& \left\langle S_{j}\right\rangle=\frac{1}{N} \sum_{k=1}^{N} S_{j}(k), \quad j=1,2,3 \\
& \left\langle S_{0}\right\rangle=S_{0},
\end{aligned}
$$

in which $S_{j}(k)$ is the $j^{\text {th }}$ Bloch component of the individual system labelled $k$. The value of $S_{0}$ is the same for all the individual systems since we consider identical two-state quantum systems. We introduce the usual vector notation: $\langle\boldsymbol{S}\rangle=\left(\left\langle S_{1}\right\rangle,\left\langle S_{2}\right\rangle,\left\langle S_{3}\right\rangle\right)$.

From the definition (51) and the Cauchy-Schwarz inequality, one finds:

$$
\left\langle S_{1}\right\rangle^{2}+\left\langle S_{2}\right\rangle^{2}+\left\langle S_{3}\right\rangle^{2} \leq\left\langle S_{0}\right\rangle^{2} .
$$


Equality is realized in (53) only in the case of the pure state, that is $\boldsymbol{S}(k)$ is a constant independent on the system $k$. We conclude that a simple criterion to know if the system is in a pure or mixed state is that the ratio $\langle\boldsymbol{S}\rangle^{2} /\left\langle S_{0}\right\rangle^{2}$ is equal to 1 if the state is pure, and less than 1 if the state is mixed.

The dynamic equations (13)-(14) for a single two-state quantum system are linear in the individual Bloch vector $\boldsymbol{S}(k)$, hence the same equations hold for the time evolution of the averaged Bloch vector $\langle\boldsymbol{S}\rangle$ which characterizes the quantum state of the ensemble of individual systems:

$$
\begin{aligned}
\frac{d\left\langle S_{0}\right\rangle}{d t} & =q(\langle\boldsymbol{S}\rangle \cdot \mathcal{E})-\Gamma S_{0}, \\
\frac{d<\boldsymbol{S}\rangle}{d t} & =q\left(\left\langle S_{0}\right\rangle \mathcal{E}+c\langle\boldsymbol{S}\rangle \times \boldsymbol{B}\right)-\Gamma\langle\boldsymbol{S}\rangle .
\end{aligned}
$$

The argument introduced in the Section 4 to unveil the equivalence with the massless charged particle, can be used here about the averaged Bloch vector. Let us define the 4-momentum, $\mathbf{P}$, of a virtual relativistic particle by:

$$
\mathbf{P} \equiv\left(\frac{E}{c}, \boldsymbol{p}\right)=e^{\bar{\Gamma} t}\left(\left\langle S_{0}\right\rangle,\left\langle S_{1}\right\rangle,\left\langle S_{2}\right\rangle,\left\langle S_{3}\right\rangle\right)
$$

with $\bar{\Gamma}$ defined in (15). If we define now the velocity of the virtual particle by:

$$
\mathbf{v} / c=\langle\boldsymbol{S}\rangle /\left\langle S_{0}\right\rangle
$$

the equations of motion (19)-(20) of the particle in the electromagnetic field $(\mathcal{E}, \mathcal{B})$ are again exactly recovered.

The only remarkable difference with the single system problem is that, now, the mass of the virtual particle is strictly positive since $\mathbf{v}^{2} / c^{2}<1$ (resulting from (53) and (57)). The probabilistic mixture of pure states makes the mass of the virtual particle to become positive.

Similar behaviour and consequences were previously noticed on an ensemble of $N$ incoherent polarized photons in [51]. This is indeed a particular case of our results on an ensemble of identical two-state quantum systems. Brosseau's analysis used the density matrix of the ensemble to make clear the relation with the dynamics of an ensemble of spin- $1 / 2$ systems.

\section{2. $N$ interacting two-state systems with non-Hermitian Hamiltonian}

Lastly, the case of an ensemble of $N$ interacting two-level systems ruled by a nonHermitian Hamiltonian may at first seem difficult to handle, but the analogy with an ensemble of MLC particles is clear. Indeed, using the results above, one can speculate that the dynamics of such an ensemble is equivalent to the collective motion of an 
ensemble of $N$ particles, each of them in the local pseudo-electromagnetic field created by all the other particles.

A standard way to solve this kind of problem is to use the mean-field approximation, that is to replace the local pseudo-electromagnetic field by its average value. This is the main approach to study the dynamics of Bose-Einstein condensates, for example [52]. Investigating this kind of collective dynamics beyond the mean-field solution, requires generally numerical simulations, such as for the dynamics of real gases (e.g. [53]) or for bird flocking (e.g. [54]).

\section{Conclusion}

We have established the unforeseen one-to-one mapping between the dynamics of a nonHermitian two-state quantum system and the motion of a massless, charged particle.

We have further exemplified such a correspondence by considering a typical twostate system in optics, namely: the evolution of light polarization when light propagates in a dichroic medium with magneto-optical effect. In this case, Faraday effect leads to the Hermitian part of the two-state system dynamics, while dichroism results in a nonHermitian part. This translates into the simple motion of a massless charged particle in a magnetic field and electric field respectively, whose values are derived explicitly from the parameters of the optical system.

Our approach has not only bridged two vastly different domains of physics, it has also paved a more intellectually-comforting way towards the understanding of intricate non-Hermitian quantum problems. We believe that the analogy drawn with the motion of a charged particle (albeit massless) in an electromagnetic field has rendered the grasping of these problems more intuitive.

\section{Acknowledgments}

R.B. and H.K. would like to thank Ritsumeikan University, BKC (Japan), and Laboratoire de Physique des Solides, Paris-Saclay (France), for their respective hospitality during preparation of this work. We are grateful to Sylvie Kwok (ESPCI) for linguistic contributions to this paper.

[1] Ke-Hsueh Li, Physics Reports 134 1-85 (1986).

[2] The Theory of Open Quantum Systems, H.-P. Breuer and F. Petruccione, Oxford University Press (2002) ; Quantum Dissipative Systems, U. Weiss, $4^{\text {th }}$ ed., World Scientific Publ., Singapore (2012).

[3] Quantum Theory of Open Systems, E. B. Davies, Academic Press, New York (1976).

[4] Open Quantum Systems. An Introduction, Á. Rivas and S. F. Huelga, SpringerBriefs in Physics (2012).

[5] I. Rotter and J. P. Bird, Reports on Progress in Physics 78114001 (2015).

[6] Y. Aharonov, S. Massar, S. Popescu, J. Tollaksen, L. Vaidman, Phys. Rev. Lett. 77, 983-987 (1996). 
[7] J. Okołowicz, M. Płoszajczak and I. Rotter, Physics Reports 374 271-383 (2003).

[8] H. Feshbach, Ann. Phys. 5357 (1958) ; H. Feshbach, Ann. Phys. 19287 (1962).

[9] I. Rotter, J. Phys. A 42153001 (2009).

[10] M. E. Brandan and G. R. Satchler, Physics Reports 285, 143-243 (1997).

[11] "Non-Hermitian Hamiltonians in Quantum Physics", F. Bagarello, R. Passante and C. Trapani eds., Springer Proceedings in Physics 184 (2016).

[12] A. Mostafazadeh, Int. J. Geom. Methods Mod. Phys. 7, 1191-1306 (2010).

[13] A. Mostafazadeh and A. Batal, J. Phys. A 37, 11645-11680 (2004).

[14] U. Günther and F. Stefani, J. Math. Phys. 443097 (2003).

[15] M. K. Oberthaler, R. Abfaleterer, S. Bernet, J. Schmiedmayer, A. Zeilinger, Phys. Rev. Lett. 77, 4980-4983 (1996), and explanation in M. V. Berry, Czechoslovak J. Phys. 54, 1039-1047 (2004).

[16] N. Moiseyev, "Non-Hermitian Quantum Mechanics", Cambridge University Press, Cambridge, 2011.

[17] G. Dattoli, A. Torre and R. Mignani, Phys. Rev. A 42, 1467-1475 (1990).

[18] D. R. Nelson, N. M. Shnerb, Phys. Rev. E 58, 1383-1404 (1998).

[19] A. Mostafazadeh, J. Math. Phys. 43, 205 (2002).

[20] for example: The Feynman Lectures on Physics - Quantum Mechanics, vol.3, R. P. Feynman, R. B. Leighton, M. Sands, chapters 8, 9, 10, 11, Addison Wesley publ. (1971) ; Quantum Mechanics, C. Cohen-Tannoudji, B. Diu, F. Laloë, 385-480, Wiley, New-York (1977).

[21] Quantum Mechanics - Non-Relativistic Theory, L. D. Landau and E. M. Lifshitz, Course of Theoretical Physics, vol.3, $2^{\text {nd }}$ ed., §132, Pergamon Press, Oxford (1965).

[22] Introduction to Quantum Mechanics, D. J. Griffiths, Prentice Hall Inc. New Jersey (1995).

[23] The 3-component vector $\mathbf{S}=\left(S_{1}, S_{2}, S_{3}\right)$ was introduced by various authors in different physical contexts. The definition used here is similar to the vector $\mathbf{r}$ introduced in: R. P. Feynman, F. L. Vernon Jr, R. W. Hellwarth, J. Appl. Phys. 28, 49-52 (1956). It is called "Bloch vector" in the context of quantum mechanics, or "Stokes vector" in the context of optics, see for example [51]

[24] see discussion and references in: R. Mignani, E. Recami, M. Baldo, Lett. Nuovo Cimento 11, 568-572 (1974), and in: I. Białynicki-Birula, Progress in Optics, XXXVI, 1-53 (1996).

[25] A. Sergi and P. V. Giaquinta, Entropy, 18, 451 (2016).

[26] K.G. Zloshchastiev and A. Sergi, J. Mod. Optics 61 1298-1308 (2014).

[27] F. Bloch, Phys. Rev. 70, 460-474 (1946).

[28] Considering the definition $\mathbf{P} \equiv(E / c, \boldsymbol{p})=\left(S_{0}, S_{1}, S_{2}, S_{3}\right)$ instead of (17), the dissipative force $-\Gamma \mathbf{v} / c$ must be added to the right-hand term of (20), and the corresponding power $-c \Gamma$ to (19).

[29] Classical Electrodynamics J. D. Jackson, Wiley (2012).

[30] "The Classical Theory of Fields", Course of Theoretical Physics, vol.2, L. D. Landau and E. M. Lifshitz, $3^{\text {rd }}$ English Edition, Pergamon Press, Oxford, 1971.

[31] K. M. Case and S. G. Gasiorowicz, Phys. Rev. 125, 1055-1058 (1962).

[32] I. Morales, B. Neves, Z. Oporto, O. Piguet, Symmetry 10, 1-15 (2018) ; Yu A. Rylov, J. Math. Phys. 30, 521-536 (1989) ; P. O. Kazinski and A. A. Shaparov, Class. Quantum Grav. 20, 2715-2725 (2003).

[33] A. Sergi and K. G. Zloshchastiev, Int. J. Mod. Phys. B27, 1350163-1350184 (2013).

[34] A. Sergi and K.G. Zloshchastiev, Physical Review A 91, 062108 (2015).

[35] A. Sergi and K.G. Zloshchastiev, J. Stat. Mech. 2016033102 (2016).

[36] A. Mostafazadeh, J. Phys. A 36, 7081-7092 (2003).

[37] Circular Dichroism and Linear Dichroism, A. Rodger and B. Nordén, §7, Oxford University Press, Oxford (1997).

[38] M. V. Berry and M. R. Dennis, Proc. R. Soc. Lond. A 459, 1261-1292 (2003).

[39] "Electrodynamics of Continuous Media", Course of Theoretical Physics, vol.8, §82, L. D. Landau and E. M. Lifshitz, $2^{\text {nd }}$ English Edition, Pergamon Press, Oxford, 1963.

[40] H. Kuratsuji and S. Kakigi, Phys. Rev. Lett. 80, 1888-1891 (1998) ; R. Botet and H. Kuratsuji, J. Phys. A 41, 035301 (2008) ; Kuratsuji, R. Botet and R. Seto, Progress of Theoretical Physics, 
117, 195-217 (2007).

[41] D. C. Brody, J. Phys. A: Math. Gen. 36, 040403 1-4 (2007).

[42] A. I. Nesterov, SIGMA 5, 069 1-17 (2009).

[43] The condition $S_{1} \rightarrow-S_{1}$ is considered in the present article, hence the coefficient $\alpha$ in (46). The formula given in [42] was written with the coefficient $\gamma$ instead of $\alpha$ because of the condition $S_{3} \rightarrow-S_{3}$ used in that article.

[44] M. Enz and R. Schilling, J. Phys. C 19, 1765-1770 (1986).

[45] for the crossed field $(\mathcal{E}, \mathcal{B})$ with $|\mathcal{E} / c \mathcal{B}|<1$, the continuous trajectory of the MLC particle with initial conditions $\mathbf{x}(0)=(0,0,0) ; \mathbf{v}(0)=(c, 0,0)$ is given by: $\mathbf{x}(t) / \xi=\log ((1+\sin \psi \sin (\Omega t-$ $\left.\left.\psi)) / \cos ^{2} \psi\right), \quad-\left(\Omega_{0} t-2(\arctan (\sin (\Omega t / 2) / \cos (\Omega t / 2-\psi))+\pi[(\Omega t / 2-\psi) / \pi+1 / 2])\right), 0\right)$, in which $[\cdots]$ denotes the integer part of the argument, and $\xi=c \cot \psi / \Omega_{0}$. The parameters $\Omega$ and $\psi$ are given by (43) and (42).

[46] A. A. Mailybaev, O. N. Kirillov and A. P. Seyranian, J. Phys. A: Math. Gen. 38, 1723-1740 (2005).

[47] H. Bacry, Annales de l'IHP 49 245-255 (1988).

[48] F. Gross, Relativistic Quantum Mechanics and Field Theory, Part II, Wiley-VCH, Weinheim (1993).

[49] As discussed in [50], two of the Maxwell equations come out from the expression of $(\mathcal{E}, \mathcal{B})$ in terms of the potentials $(\Phi, \mathbf{A})$, whereas the remaining two Maxwell equations may be regarded as definitions of the charge density and of the current density.

[50] F. J. Dyson, Am. J. Phys. 58, 209-211 (1990) ; N. Dombey, Am. J. Phys. 59, 85-85 (1991) ; R. W. Brehme, Am. J. Phys. 59, $85-86$ (1991) ; J. L. Anderson, Am. J. Phys. 59, 86-86 (1991) ; I. E. Farquhar, Am. J. Phys. 59, 87-87 (1991) ; Z.K. Silagadze, Annales Fond. Broglie 27 241-256 (2002).

[51] Fundamentals of Polarized Light. A Statistical Optics Approach, C. Brosseau, John Wiley and Sons, New York (1998).

[52] J. Kronjäger, C. Becker, M. Brinkmann, R. Walser, P. Navez, K. Bongs and K. Sengstock, Phys. Rev. A 72, 063619 (2005).

[53] The Art of Molecular Dynamics Simulation, D. C. Rapaport, Cambridge University Press (1996).

[54] W. Bialek, A. Cavagna, I. Giardina, T. Mora, E. Silvestri, M. Viale, and A. M. Walczak, PNAS 109, 4786-4791 (2012). 